Bangladesh J. Plant Taxon. 15(2): 129-139, 2008 (December)

(C) 2008 Bangladesh Association of Plant Taxonomists

\title{
A TAXONOMIC ACCOUNT ON THE PHYTOPLANKTON OF A POND RECEIVING TEXTILE INDUSTRIAL EFFLUENTS
}

\author{
Z.N. TAHMIDA BEGUM ${ }^{1}$ \\ Department of Botany, University of Dhaka, Dhaka 1000, Bangladesh
}

Keywords: Industrial effluents, Phytoplankton, Taxonomy

\begin{abstract}
Phytoplankton from four stations of a large pond receiving effluents from two textile industries have been investigated. A total of 69 taxa were identified out of which 48 belonged to Chlorophyceae followed by 17 to Cyanophyceae. One species from each of Chrysophyceae, Xanthophyceae, Cryptophyceae and Dinophyceae were also recorded. Oscillatoria limnetica Lemm., Anabaena circinalis Rabenh. ex Born. et Flah., Nostoc commune Vaucher ex Born. et Flah. Ankistrodesmus falcatus var. mirabilis (West \& West) Lemm. and Scendesmus spp. were found dominant in all the stations.
\end{abstract}

\section{Introduction}

The effluents, discharged in rivers, ponds, lakes, etc., are as varied as the human activities which produce them. According to Hynes and Pentelow (1978) six categories of effluents exist, namely (i) inert suspensions, (ii) poisons, (iii) inorganic reducing agents, (iv) oils, (v) organic residues, and (vi) hot water. The degree of pollution can often be measured most easily by a biological analysis in which phytoplankton are important indicators. For the assessment of water quality biological indicators are better than chemical and physical features (Round 1985). In India, aspects of biology of industrial wastewater have been studied by Mohan and Kumar (1990) and Baliarsingh et al. (1991).

In Bangladesh, a number of research works have been carried out on phytoplankton from a range of habitats and localities (Islam and Begum 1970, 1987, Islam and Irfanullah 2005a, b, c, 2006, Khondker et al. 2007a, b). Phytoplankton from organically polluted ponds were worked out by Islam and Khatun (1966), Islam and Nahar (1967), and Khondker et al. (1990), but there exists very little information on qualitative aspects of phytoplankton from the polluted habitats contaminated by industrial wastes. The present work has therefore been undertaken to study qualitative account of phytoplankton (excluding members of Bacillariophyceae and Euglenophyceae) from a pond receiving wastewater from two textile industries in Dhaka.

\section{Materials and Methods}

The investigation was carried out in a large permanent pond near Deilla, Demra, Dhaka during 1990-1991. The pond is an open drainage type having one inlet and is regularly charged with effluent wastewater released from the nearby textile and dyeing

$\overline{{ }^{1} \text { E-mail: botany@univdhaka.edu }}$ 
industries. Four stations or sampling points, namely S-1, S-2, S-3 and S-4, were selected in the pond for sampling.

Samples were collected at fortnight intervals at a depth of about $25 \mathrm{~cm}$ below the surface of water. A rafter was used for this purpose. Air and water temperatures were recorded by a mercury (centigrade) thermometer. $\mathrm{pH}$ and redox potential (RP) were measured by CD-300 digital portable $\mathrm{pH}$ meter with the help of the electrode (PHMHBA-220U, 24:760/6). Dissolved oxygen (DO) and free carbon dioxide were analyzed according to APHA (1976). The bicarbonate alkalinity was measured after Gerrath and Denny (1979).

Collection and preservation of samples for biological analysis were done according to Johansen (1940) and Khondker et al. (1990). Phytoplankton were identified with the help of a Nikon compound microscope (Japan) at magnifications ranging from $\times 150-\times 1500$. Desikachary (1959), Islam and Begum (1970), Prescott (1982), Huber-Pestalozzi (1983), Bold and Wynne (1985), Islam and Irfanullah (2005a, b, c) and Khondker et al. (2006, 2007a, b) were consulted for the identification of phytoplankton species.

\section{Results and Discussion}

Table 1 shows annual ranges of some physicochemical variables from the studied pond. Except S-1 no significant variation was observed in case of air and water temperatures. Annual maximum water temperature recorded at this station was about 6$7^{\circ} \mathrm{C}$ higher compared to the maximum temperature recorded in other stations. Might be this station was receiving some hot water discharge from the industries. A minimum $\mathrm{pH}$ value has been recorded at this station and S-4. Anoxia was observed in all the stations. Carbon dioxide was occasionally undetectable at S-1 and S-3. Similarly bicarbonate alkalinity was also undetectable at different times in all the stations.

Table 1. Range of some physical and chemical variables from four stations of the pond in Dhaka, 1990-1991.

\begin{tabular}{lccccccc}
\hline $\begin{array}{l}\text { Sampling } \\
\text { station }\end{array}$ & $\begin{array}{c}\text { Air } \\
\text { temperature } \\
\left({ }^{\circ} \mathrm{C}\right)\end{array}$ & $\begin{array}{c}\text { Water } \\
\text { temperature } \\
\left({ }^{\circ} \mathrm{C}\right)\end{array}$ & $\mathrm{pH}$ & $\begin{array}{c}\text { Dissolved } \\
\text { oxygen } \\
(\mathrm{mg} / \mathrm{l})\end{array}$ & $\begin{array}{c}\text { Free } \\
\mathrm{CO}_{2} \\
(\mathrm{mg} / \mathrm{l})\end{array}$ & $\begin{array}{c}\text { Bicarbonate } \\
\text { alkalinity } \\
(\mathrm{meq} / \mathrm{l})\end{array}$ & $\begin{array}{c}\text { Redox } \\
\text { potential }\end{array}$ \\
\hline $\mathrm{S}-1$ & $23-38$ & $20-39$ & $5-7$ & $0-5$ & $0-8$ & $0-19$ & $-0.43-0.29$ \\
$\mathrm{~S}-2$ & $23-36$ & $20-32$ & $6-7$ & $0-6$ & $1-6$ & $0-8$ & $-0.21-0.29$ \\
$\mathrm{~S}-3$ & $22-36$ & $20-33$ & $6-7$ & $0-5$ & $0-9$ & $0-19$ & $-0.19-0.29$ \\
$\mathrm{~S}-4$ & $23-36$ & $18-32$ & $5-7$ & $0-5$ & $1-9$ & $0-5$ & $-0.22-0.27$ \\
\hline
\end{tabular}

A total of 69 phytoplankton taxa were recorded from the pond. The taxa which were found to be dominant were also previously reported to be dominant in different aquatic habitats in Bangladesh. The taxa belonged to the classes Cyanophyceae, Chlorophyceae, Chrysophyceae, Xanthophyceae, Cryptophyceae and Dinophyceae. A brief account on each taxon has been provided below including its abundance in station(s). 


\section{Class: Cyanophyceae; Order: Chroococcales; Family: Chroococcaceae}

1. Aphanothece pallida Kütz. (Rebenh.), Fl. Eur. Alg. 2: 64 (1865). Colony 4-6 $\mu \mathrm{m}$ broad; cells 7-9 broad, 14-16 $\mu \mathrm{m}$ long with sheath, without sheath 6.5 broad, 6.5-10.0 $\mu \mathrm{m}$ long. Stations: 1-4; common.

\section{Order: Oscillatoriales; Family: Oscillatoriaceae}

2. Oscillatoria agardhii Gomont, Monogr. Oscillariées, 205 (1892). Filamentous; cells 2.0-6.6 broad, 1-3 $\mu \mathrm{m}$ long; calyptra 2.5 broad, $1.8 \mu \mathrm{m}$ long. Stations: 2-4; few.

3. Oscillatoria amphibia Ag. ex Gomont, Monogr. Oscillarièes, 221, pl. 7, figs 4-5 (1892). Tip cell $3 \mu \mathrm{m}$ broad, $13 \mu \mathrm{m}$ long; individual cell $5 \mu \mathrm{m}$ broad, $3 \mu \mathrm{m}$ long. Station: 3; rare.

4. Oscillatoria homogenea Frémy, Myxo. d’ Afr. Équat. Franc. 215, fig.184 (1929). Trichomes long; cells 5.7-8.5 $\mu \mathrm{m}$ broad, 4-7 $\mu \mathrm{m}$ long. Stations: 1-4; common.

5. Oscillatoria limnetica Lemm., Ber. dtsch. Bot. Ges. 18: 310 (1900). [Syn.: O. splendida var. limnetica (Lemm.) Playfair]. Trichomes straight or bent; cells 1.5-2.0 $\mu \mathrm{m}$ broad, 2.5-5.0 $\mu \mathrm{m}$ long. Stations: 1-4; very common.

6. Oscillatoria pseudogeminata G. Schmid., Ber. dtsch. Bot. Ges. 32: 124, fig. 4 (1914). Trichomes straight; individual cell $3 \mu \mathrm{m}$ broad, $8 \mu \mathrm{m}$ long; terminal cell 2-3 $\mu \mathrm{m}$ broad, 4-8 $\mu \mathrm{m}$ long. Stations: 1-4; common.

7. Oscillatoria sancta (Kütz.) Gomont, Monogr. Oscillariées, 209, pl. 6, fig. 12 (1892). Thallus composed of many trichomes; cells 9.2-16.0 $\mu \mathrm{m}$ broad, 2.3-6.1 $\mu \mathrm{m}$ long. Stations: 1, 3, 4; few.

8. Oscillatoria subbrevis Schmidle., Engler's Bot. Jahrb. 30; 243, pl. 4, fig. 7 (1901). Trichomes single, 5-6 $\mu \mathrm{m}$ broad; cells 1-2 $\mu \mathrm{m}$ long. Stations: 1-3; few.

9. Spirulina gigantea Schmidle., Engler’s Bot. Jahrb. 32: 59, pl. 1, fig. 5 (1902). Trichomes 2.7-4.0 $\mu \mathrm{m}$ broad, regularly spirally coiled, spirals 8-16 $\mu \mathrm{m}$ broad. Station: 1 ; rare.

\section{Family: Nostocaceae}

10. Anabaena affinis Lemm., Zeit. f. Fisch. 1897: 177-188 (1897). Filaments long; cells 6.0-7.5 $\mu \mathrm{m}$ broad, 4.0-4.5 $\mu \mathrm{m}$ long; heterocysts 6.5-8.5 $\mu \mathrm{m}$ broad, 5.5-6.2 $\mu \mathrm{m}$ long. Stations: 1-4; common.

11. Anabaena circinalis Rabenh. ex Born. et Flah., Algen Eur. Exs. no. 209 (1852). [Syn.: A. flos-aquae var. circinalis Kirchner]. Trichome 5-8 $\mu \mathrm{m}$ broad; cells 7-10 $\mu \mathrm{m}$ 
long, 5-6 $\mu \mathrm{m}$ broad; heterocysts sub-spherical, 7-11 $\mu \mathrm{m}$ broad. Stations: 1-4; very common.

12. Anabaena flos-aquae (Lyngb.) Bréb. ex Born. et Flah. in Bréb. et Godey, Algues des environs de Falaise, 36 (1835). Filamentous; cells 5-6 $\mu \mathrm{m}$ broad; heterocysts 6-7 $\mu \mathrm{m}$ broad, $7.8 \mu \mathrm{m}$ long. Stations: 1-4; common.

13. Anabaena naviculoides Fritsch, J. Indian Bot. Soc. 28: 138, figs 17-39 (1949). Trichomes elongate, coiled; cells 3.5-5.0 $\mu \mathrm{m}$ broad. Station: 1 ; very rare.

14. Anabaena orientalis Dixit, Proc. Indian Acad. Sci. B, 3: 101, fig. 3 D, E (1936). Trichomes single, $6.5 \mu \mathrm{m}$ broad; cells 3.6-7.0 $\mu \mathrm{m}$ broad, 5-7 $\mu \mathrm{m}$ long; heterocysts 5$7 \mu \mathrm{m}$ broad, 7.0-11.2 $\mu \mathrm{m}$ long; akinetes 11-14 $\mu \mathrm{m}$ broad, 18.1-21.5 $\mu \mathrm{m}$ long. Stations: 3, 4; not very common.

15. Anabaena volzii Lemm., Abh. Nat. Ver. Bremen, 18: 153, pl. 9, figs 4, 5, 20 (1906). [Syn.: Anabaena unispora Gardner]. Filamentous; cells 3.6-4.8 $\mu \mathrm{m}$ broad, 7-10 $\mu \mathrm{m}$ long; heterocysts 3.6-8.0 $\mu \mathrm{m}$ broad, 7-16 $\mu \mathrm{m}$ long; akinetes 9.6-20.0 $\mu \mathrm{m}$ broad, 20-34 $\mu \mathrm{m}$ long. Stations: 1-4; few.

16. Anabaenopsis raciborskii Wolosz., Bull. Int. Acad. Sci. Cracovie, B, 6: 684, fig. 10 (1913). Filaments 40-130 $\mu \mathrm{m}$ long; cells 2-3 $\mu \mathrm{m}$ broad, 4.5-11.0 $\mu \mathrm{m}$ long; heterocysts 3.0-3.5 $\mu \mathrm{m}$ broad, 5.5-7.5 $\mu \mathrm{m}$ long; akinete-like structure 4.0-4.3 $\mu \mathrm{m}$ broad, 7-9 $\mu \mathrm{m}$ long. Station: 2; very rare.

17. Nostoc commune Vaucher ex Born. et Flah., Historoie des conferves d'eau douce, 222, pl. 16, fig. 1 (1803). Colony $84 \mu \mathrm{m}$ broad, $183 \mu \mathrm{m}$ long; cells $4-5 \mu \mathrm{m}$ broad, 5-7 $\mu \mathrm{m}$ long; heterocysts $5 \mu \mathrm{m}$ broad, $3 \mu \mathrm{m}$ long. Stations: 1-4; very common.

\section{Class: Chlorophyceae; Order: Volvocales; Family: Volvocaceae}

18. Eudorina elegans Ehrenberg, Monatsber. Akad. Wiss. Berlin 183: 78, 152 (1833). Colony 32-celled (also 16-celled), 69-96 $\mu \mathrm{m}$ broad, c 60-200 $\mu \mathrm{m}$ long; cells 7.0-11.7(25.0) $\mu \mathrm{m}$ broad; pyrenoids 3 to many. Station: 1 ; rare.

19. Pandorina morum (Müller) Bory, Encycl. Meth. Diet. Hist. Nat., p. 600 (1824). Colony 8-16-(rarely-32)-celled, 20-33(-42) $\mu \mathrm{m}$ broad, 30-41(-60) $\mu \mathrm{m}$ long (may be longer); cells 6.6-10.0(-17.0) $\mu \mathrm{m}$ broad, 9-13(-17) $\mu \mathrm{m}$ long; flagella 2, 2.0-2.5 times body length; contractile vacuole 2 . Stations: 2 , 3; few.

\section{Order: Chlorococcales; Family: Chlorococcaceae}

20. Schroederia setigera (Schröd.) Lemmermann, Hedwigia, Dresden, 37: 303-312 (1898). [Syn.: Reinschiella setigera Schröd., Ankistrodesmus setigerus (Schröd.) G.S. 
West, Characium setigerum (Schröd.) Bour.]. Cells $4.4 \mu \mathrm{m}$ broad, $105.6 \mu \mathrm{m}$ long with spines. Stations: 2-4; rare.

\section{Family: Hydrodictyaceae}

21. Pediastrum duplex Meyen, Nova Acta Loep. Carol., Norimberge, 14(2): 768-778 (1829). [Syn.: P. napoleonis Ralfs, P. pertusum Kützing]. Colony 8-128-celled, up to $182 \mu \mathrm{m}$ broad; cells 10.0-16.2 $\mu \mathrm{m}$ broad, 13.5-21.78 $\mu \mathrm{m}$ long. Stations: 1 , 2; few.

22. Pediastrum duplex var. gracillimum West \& West, J. Roy. Microsc. Soc., London, 14: 1-17 (1894). [Syn.: P. gracile A. Br., P. gracillimum Thun.]. Colony 4-32-celled; cells 2.3-13.2 $\mu \mathrm{m}$ broad, 5.5-13.2 $\mu \mathrm{m}$ long. Station: 2; rare.

23. Pediastrum duplex var. reticulatum Lagerheim, Ofv. Kgl. Sv. Vet.-Akad. Forh. 39: 47-81 (1882). [Syn.: P. duplex var. clathratum (Ag. Br.) Lagerheim]. Colony 8-32celled; outer cells 4.4-20.0 $\mu \mathrm{m}$ broad, 8.8-27.0 $\mu \mathrm{m}$ long; inner cells 2.3-17.6 $\mu \mathrm{m}$ broad, 6.6-20.55 $\mu \mathrm{m}$ long. Stations: 2-4; few.

24. Pediastrum tetras (Ehrenberg) Ralfs, Ann. \& Mag. Nat. Hist. 14: 469 (1844). [Syn.: P. rotula Kütz., P. ehrenbergii (Chodat) A. Braun, P. incavatum Turn.]. Colony 4-8celled; inner cells with 4-6 straight sides, cells 4.4-6.6 $\mu \mathrm{m}$ broad, 5.5-9.0 $\mu \mathrm{m}$ long. Stations: 2-4; common.

25. Pediastrum tetras var. tetraedron (Corda) Hansgirg, Prod. Algen. Böhmen-1. Teil, 288 pp. Prag (1886). Colony 4-celled, 21.3-40.0 $\mu \mathrm{m}$ long; cells 2.2-13.3 $\mu \mathrm{m}$ broad, 7.26-14.2 $\mu \mathrm{m}$ long. Stations: 1 , 4; very rare.

\section{Family: Oocystaceae}

26. Ankistrodesmus falcatus (Corda) Ralfs, Ann. Bot. 34: 49, 74, fig. 2 (1848). [Syn.: Micrasterias falcata Corda, A. biplex (Reinsch) G.S. West, A. lundbergii Kors.]. Cells 1.1-2.0 $\mu \mathrm{m}$ broad, 30.2-66.0 $\mu \mathrm{m}$ long, solitary or in clusters of 2-32 individuals. Stations: 1-4; very common.

27. Ankistrodesmus falcatus var. mirabilis (West and West) Lemmermann (1908). Cells $2 \mu \mathrm{m}$ broad, 19.8-37.4 $\mu \mathrm{m}$ long; autospores 4-8 in number. Stations: 1-4; very common.

28. Ankistrodesmus spiralis (Turner) Lemmerman, Ark. Bot. Kristiania 2: 1-209 (1904). [Syn.: Raphidium spirale Turn., Raphidium polymorphum Fres., Raphidium turneri Bern.]. Colony 2-8 or more celled; cells 1.1-2.2 $\mu \mathrm{m}$ broad, 25.8-30.8 $\mu \mathrm{m}$ long; autospores 4-8 in number. Stations: 2, 4; rare. 


\section{Family: Scenedesmaceae}

29. Crucigenia lauterbornii (Schmidle) Schmidle, Allg. Bot. Z., Karlsruhe, 5: 2-4 (1900). [Syn.: Hofmannia lauterbornii (Schmidle) Wille, Komarenkia lauterbornii (Schmidle) Fott, Staurogenia lauterbornii Schmidle]. Colony 4-celled; cells 4.4-6.6 $\mu \mathrm{m}$ broad, 6.6-6.7 $\mu \mathrm{m}$ long. Station: 1; rare.

30. Crucigenia quadrata Morren, Ann. Sci. Nat. (a). Paris, 20: 404-426 (1830). [Syn.: Micrasterias crucigenia Kütz., Staurogenia quadrata (Morr.) Kütz.]. Colony 4celled, forming 16-celled multiple coenobia; colony 13.2-36.0 $\mu \mathrm{m}$ in diameter; cells 3.3-6.6 $\mu \mathrm{m}$ broad, 3.3-9.9 $\mu \mathrm{m}$ long; chloroplasts as many as 4 in a cell. Stations: 1 , 2; few.

31. Crucigenia tetrapedia (Kirchner) West and West, Trans-Roy. Irish Acad. 32 (B): 1100 (1902). [Syn.: Staurogenia tetrapedia Kirchner, Tetrapedia kirchneri Lemm., Lemmermannia tetrapedia (Kirchn.) Lemm.]. Colony 4-celled, forming 16-celled multiple coenobia; colony $6.6 \mu \mathrm{m}$ in diameter; cells triangular, 2.2-4.4 $\mu \mathrm{m}$ broad, 6.4-6.7 $\mu \mathrm{m}$ long. Stations: 1, 2; few.

32. Crucigeniella crucifera (Wolle) Komárek, Arch. Protistenk., Jena, 116: 1-75 (1974). [Syn.: Staurogenia crucifera Wolle, Crucigenia cruciata Schmidle, C. crucifera (Wolle) Collins]. Colony 4-16-celled, 8.8-11.0 $\mu \mathrm{m}$ broad, 8.8-14.2 $\mu \mathrm{m}$ long, cells 2.2-6.6 $\mu \mathrm{m}$ broad, 4.4-10.0 $\mu \mathrm{m}$ long. Station: 2; rare.

33. Crucigeniella rectangularis (Näg.) Komárek, Arch. Protistenk., Jena, 116: 1-75 (1974). [Syn.: Staurogenia rectangularis Nägeli in ex Braun, Crucigenia rectangularis (Nägeli) Gay]. Colony 4-32-celled; cells 2.2-3.5 $\mu \mathrm{m}$ broad, 2.4-6.6 $\mu \mathrm{m}$ long; chloroplasts 1-4 parietal discs. Stations: 1-4; common.

34. Scenedesmus acutiformis Schöder, Ber. Dt. Bot. Ges., Stuttgart 15: 372-373 (1897). [Syn.: S. quadricauda var. acutiformis (B. Schröder) Schmidle, S. hystrix var. acutiformis (B. Schröder) R. Chodat, S. hystrix f. acutiformis (B. Schröder) R. Volk]. Colony 4-celled; cell $6 \mu \mathrm{m}$ long. Stations: 1, 2, 4; few.

35. Scenedesmus arcuatus Lemmermann, Forschungsber. Biol. Stat. Plön 7: 96-135 (1899). Colony 4-16-celled; cells 4.4-8.8 $\mu \mathrm{m}$ broad, 6.6-14.3 $\mu \mathrm{m}$ long. Stations: 1-4; very common.

36. Scenedesmus arcuatus var. platydiscus G.M. Smith, Trans. Wisc. Acad. Sci. Arts and Letters, Madison, 18: 422-539 (1916). Colony 4-8-celled, $11.0 \mu \mathrm{m}$ long; cells oblong-elliptic, 2.2-6.6 $\mu \mathrm{m}$ broad, 6.8-8.8 $\mu \mathrm{m}$ long. Stations: 1, 3; few.

37. Scenedesmus bijuga (Turp.) Lagerheim, Nuora Notarisia 2: 153-191 (1893). [Syn.: S. helveticus Chodat]. Colony 2-4-8-celled, $13.5 \mu \mathrm{m}$ long; cells 3.3-10.0 $\mu \mathrm{m}$ broad, 8.8-20.0 $\mu \mathrm{m}$ long. Stations: 1-4; common. 
38. Scenedesmus bijuga var. irregularis (Wille) G.M. Smith, Trans. Wisc. Acad. Sci. Arts and Letters, Madison 18: 422-539 (1916). Colonial; cells 3.3-6.6 $\mu \mathrm{m}$ broad, 6.6$8.8 \mu \mathrm{m}$ long. Stations: 1, 3, 4; common.

39. Scenedesmus brasiliensis Bohlin, Bih. K. Svenska Vet.-Akad. Handl. 23, Afd. 3, No. 7: 3-47 (1897). Colony 2-4-8-celled, arranged in a single series, 21-27 $\mu \mathrm{m}$ long; poles with 1-2 small teeth; cells 4.4-7.4 $\mu \mathrm{m}$ broad, 11.0-22.3 $\mu \mathrm{m}$ long. Stations: 1 , 4; rare.

40. Scenedesmus denticulatus Lagerheim, Öfv. Kongl. [Svenska] Vet.-Akad. Förh. 39(2): 47-81 (1882). Colony 4-8-celled, arranged in a single series; cells 3.3-10.0 $\mu \mathrm{m}$ broad, 11.0-20.9 $\mu \mathrm{m}$ long; 1-4 short teeth/spines at cell apices; spines $3.3 \mu \mathrm{m}$ long. Stations: 1-4; common.

41. Scenedesmus dimorphus (Turp.) Kütz., Linnaea 8: 604-609 (1833). [Syn.: Achnanthes dimorpha Turp., S. pectinatus Meyen, S. acutus var. dimorphus (Turp.) Rabenh.]. Colony 4-8-celled; cells 2.2-10.0 $\mu \mathrm{m}$ broad, 11-23 $\mu \mathrm{m}$ long. Stations: 1-4; common.

42. Scenedesmus incrassatulus Bohlin, K. Svenska Vet.- Akad. Handl. Stockholm, Afd.

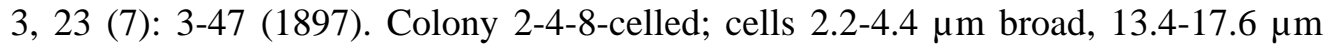
long. Stations: 2-4; few.

43. Scenedesmus magnus Meyen, Nova Acta Leop. Carol., Norimbergae 14(2): 768-778 (1829). [Syn.: S. longus var. naegelli Bréb., S. longus Meyen, S. quadricauda Bréb. var. maximus (West \& West) Chodat.]. Colony flat, 2-4-8-celled; cells 3.0-8.8 $\mu \mathrm{m}$ broad, 7-23 $\mu \mathrm{m}$ long. Stations: 1, 2, 4; few.

44. Scenedesmus longus var. brevispina G.M. Smith, Trans. Wisc. Acad. Sci. Arts. Lett., Madison 18: $422-530$ (1916). Colonial; cells 4.5-6.6 $\mu \mathrm{m}$ wide, 15.4-17.4 $\mu \mathrm{m}$ long; setae $2.2 \mu \mathrm{m}$ long. Stations: 3, 4; rare.

45. Scenedesmus perforatus Lemmermann, Forschungsber. Biol. Stat. Plön 11: 289-311 (1904). Colony 2-8-celled; cells 3.0-8.8 $\mu \mathrm{m}$ broad, 9.9-26.4 $\mu \mathrm{m}$ long; setae 2.2-15.4 $\mu \mathrm{m}$ long. Station: 1 ; very rare.

46. Dictyosphaerium ehrenbergianum Nägeli, Gattungen einzelliger Algen. pp. 137, Zürich (1849). Colony $85 \mu \mathrm{m}$ in diameter; individual cells 3.3-6.6 $\mu \mathrm{m}$ broad, 6.6-9.9 $\mu \mathrm{m}$ long. Stations: 1-4; very common.

47. Hyaloraphidium contortum Pascher \& Korsikov, Arch. Protistenk. 74: 249, figs 1-6 (1931). Cells 2.2-2.5 $\mu \mathrm{m}$ broad, 19.8-28.6 $\mu \mathrm{m}$ long. Stations: 1-4; common.

48. Kirchneriella lunaris (Kirch.) Moebius, Abh. Senck. Natur. Ges. Frankfurt A.M. 18: 309-350 (1894). [Syn.: Raphidium convolutum var. lunare Kirchn., K. lunata 
Schmidle]. Colony 4-16-celled, up to $48.4 \mu \mathrm{m}$ in diameter; cells 4.4-6.6 $\mu \mathrm{m}$ broad, 3.3-9.9 $\mu \mathrm{m}$ long. Station: 2; rare.

49. Trochiscia reticularis (Reinsch) Hansgirg, Hedwigia, 27: 126-132 (1888). Cells usually in clumps, 26.4-33.0 $\mu \mathrm{m}$ in diameter; autospores 4 , 8 or 16. Stations: 1 , 2; not very common.

50. Tetraedron regulare Kützing, Phycologia germanica, d.i. Deutschlants Algen in bündigen Beschreibungen, pp. 340. Nordhausen (1845). [Syn.: T. tumidulum (Reinsch) Hansgirg, T. quadrilobum G.M. Smith]. Cells tetragonal, $50.7 \mu \mathrm{m}$ broad (with spine), 15.5-57.4 $\mu \mathrm{m}$ long. Stations: 1, 2; rare.

51. Tetraedron trigonum (Nägeli) Hansgirg, Hedwigia, 27: 126-132 (1888). Cells 8.6$22.0 \mu \mathrm{m}$ broad, $11.2-22.0 \mu \mathrm{m}$ long. Stations: 1-4; common.

52. Tetraedron constrictum G.M. Smith, Wis. Geol. and Nat. Hist. Surv., Bull. 57: 1-243 (1920). Cells $28.6 \mu \mathrm{m}$ in diameter with processes. Station: 2; very rare.

53. Tetraedron caudatum (Corda) Hansgirg, Hedwigia, Dresden, 27 (516): 126-132 (1888). [Syn.: Polyedrium pentagonum Reinsch]. Cell diameter $8.8 \mu \mathrm{m}$; autospores 2-4-8 per cell. Stations: 1, 2, 4; few.

\section{Family: Coelastraceae}

54. Actinastrum hantzschii Lagerheim, Öfv. Kongl. Sv. Vet.-Akad. Förhandl, 39 (2): 47-81 (1882). [Syn.: Ourococcus bicaudatus (A. Braun) Grobety]. Colony 4- or 8celled; cells 2.2-4.2 $\mu \mathrm{m}$ broad, 8.8-19.6 $\mu \mathrm{m}$ long. Stations: 1-4; very rare.

\section{Order: Zygnematales; Family: Desmidiaceae}

55. Closterium moniliferum (Bory) Ehrenberg, Infusions. Volkomm. Organism. p. 91, pl. 5, fig. 16; ex Ralfs 1848, Brit. Desm. 166, pl. 28: 3 (1838). [Syn.: C. leibleinii Kg. ex Ralfs proparte, C. malinvernianiforme Groenblad, C. moniliferum (Bory) Her. ex Ralfs var. malinvernianiforme (Groenb.) Kosinsk.]. Cells solitary, 166-261 $\mu \mathrm{m}$ long, median diameter 29-45 $\mu \mathrm{m}$, apex (3)-6-9 $\mu \mathrm{m}, 50^{\circ}-133^{\circ}$ arc; chloroplast 5-10, pyrenoids 4-10; terminal vacuole with c 10 granules. Station: 2; very rare.

56. Closterium ralfsii Bréb. ex Ralfs var. gracilius (Maskell) Krieger, Rabenhorst's Kryptog. Flora 13: 346, pl. 31, fig. 6 (1937). [Syn.: C. decorum Bréb. var. gracilius Maskell]. Cell length $210 \mu \mathrm{m}$, median diameter $11 \mu \mathrm{m}$, apex $3 \mu \mathrm{m}$, curvature less, c $30^{\circ}$ arc; striation 5-14 in $10 \mu \mathrm{m}$. Station: 4; very rare.

57. Cosmarium caelatum Ralfs, Brit. Desmid.: 103 (1848). Cells $18.2 \mu \mathrm{m}$ broad, 21.8$23.2 \mu \mathrm{m}$ long, isthmus 3.6-5.4 $\mu \mathrm{m}$, apex $10 \mu \mathrm{m}$. Station: 2; rare. 
58. Cosmarium impressulum Elfving, Acta Soc. Fauna Flora Fenn. 2 (2): 13, pl.1, fig. 9 (1881). [Syn.: C. meneghinii var. simplicissimum f. reinschii Istvanfy]. Cells 18-26 $\mu \mathrm{m}$ long, 12.6-18.5 $\mu \mathrm{m}$ broad, isthmus 3.6-7.4 $\mu \mathrm{m}$, apex 5.5-7.3 $\mu \mathrm{m}$. Station: 3; rare.

59. Cosmarium laeve Rab., F1. Eur. Aig. 3: 161 (1858). Cells $25.4 \mu \mathrm{m}$ long, median diameter at the base of semicell 7.5-14.5 $\mu \mathrm{m}$, isthmus 3.3-6.9 $\mu \mathrm{m}$. Stations: 2-4; common.

60. Staurastrum galeatum Turner, Alg. Ind. Orient.: 122, pl. 14, figs 3, 9-10 (1893). Cells 29.6-30.0 $\mu \mathrm{m}$ long, median diameter with processes 37-39 $\mu \mathrm{m}$, isthmus $11 \mu \mathrm{m}$. Station: 4; rare.

61. Staurastrum lapponicum (Schmidle) Grönblad, Soc. Sc. Fenn., cimment. Biol. 2(5): 29 (1926). [Syn.: S. punctulatum var. muricatiforma fa. lapponica Schmidle]. Cells $25 \mu \mathrm{m}$ long, median diameter 23-24 $\mu \mathrm{m}$, isthmus $8.3 \mu \mathrm{m}$. Station: 4; rare.

62. Staurastrum longibrachiatum West \& West, Nova Hedwigia pl. 17, figs 8, 9 (1905). [Syn.: S. bicorne var. longebrachiatum Borge]. Cell length without processes 26-46 $\mu \mathrm{m}$, median diameter with processes 60-90 $\mu \mathrm{m}$, isthmus 8-10 $\mu \mathrm{m}$. Station: 4; rare.

63. Staurastrum manfeldtii Delponte, Hirano, Mem. R. Accad. Sc. Torino, ser. 2, 30: 64 (1878). Cell length $35 \mu \mathrm{m}$, median diameter without spines $13.6 \mu \mathrm{m}$, isthmus 8.3 $\mu \mathrm{m}$. Stations: 1, 2, 4; few.

64. Pleurotaenium trabecula (Her.) Nägeli., Gattung einz. Algen, 104, pl. 6, fig. A (1849). Cells $576 \mu \mathrm{m}$ long, median diameter at the base of semicell $50 \mu \mathrm{m}$, isthmus $42 \mu \mathrm{m}$, cell apex 25.0-33.4 $\mu \mathrm{m}$. Stations: 3, 4; rare.

65. Euastrum spinulosum Delponte var. inermius (Nordstedt) Bernard, p. 126, pl. 8, figs 207, 208 in Protococcácées et Desmidiées d'eau douce, recoltees á Java, pp. 230, Batavia (1908). [Syn.: E. spinulosum Delp. subsp. inermius Nordstedt]. Cells 49-59(81) $\mu \mathrm{m}$ long, median diameter 42-51-(67) $\mu \mathrm{m}$, isthmus 8.5-11-(18) $\mu \mathrm{m}$, apex 12.517-(27) $\mu \mathrm{m}$. Station: 4; rare.

\section{Class: Chrysophyceae; Sub-class: Chrysophycidae; Order: Ochromonadales; Family: Dinobryaceae}

66. Dinobryon sertularia Ehrenberg, Abh. K. Aked. Wiss. Berlin, Physik. K1. 1833: 280 (1834). Lorica $9.4 \mu \mathrm{m}$ broad, 32.4-36.4 $\mu \mathrm{m}$ long, opening diameter $10.8 \mu \mathrm{m}$; zygospore diameter $14.8 \mu \mathrm{m}$. Stations: 2-4; not so common. 


\section{Class: Xanthophyceae; Order: Mischococcales; Family: Pleurochloridaceae}

67. Isthmochloron gracile (Reinsch) Skuja var. dacchense Islam, Dacca Univ. Stud. 21, pt. B. (1973). Cells solitary, with arms 22-34 $\mu \mathrm{m}$ broad, 28.8-34.0 $\mu \mathrm{m}$ long. Stations: 2-4; not so common.

\section{Class: Dinophyceae; Order: Peridiniales; Family: Peridiniaceae}

68. Ceratium hirundinella (Müller) Dujardin, Infusoires: 377 (184l). [Syn.: Bursarja hirundinella Müller]. Cell proper $41 \mu \mathrm{m}$ broad, $150 \mu \mathrm{m}$ long; epicone with horn 95 $\mu \mathrm{m}$ long; hypocone with posterior horn $64 \mu \mathrm{m}$ long. Stations: 1, 2; not so common.

\section{Class: Cryptophyceae; Order: Cryptomonadales; Family: Cryptomonadaceae}

69. Cryptomonas obovata Skuja, Acta Horti Bot. Univ. Latv. 11-12: 41-169 (1939). Cells $13 \mu \mathrm{m}$ broad, $25 \mu \mathrm{m}$ long; flagella 2, equal or unequal, $12 \mu \mathrm{m}$ long. Stations: $2-$ 4; common.

\section{Acknowledgements}

The author is grateful to $\mathrm{Md}$. Zahangir Hossain for the assistance during the collection of the materials. Thanks are also due to two textile mill authorities for permitting the author to sample their pond.

\section{References}

APHA, 1976. Standard methods for the examination of water and waste water (14th ed.). American Public Health Association, Washington, pp. 1-1193.

Baliarsingh, P.K., Routray, B., Chowdhury, R.C. and Padhi, S. 1991. Effect of environmental factors on plankton community in various effluent receiving sites of sugar industry of Aska (Orissa). In: Islam, A.K.M. Nurul, Fattah, Q.A., Muttaqi, I.A. and Aziz, A. (eds), Plant Science and Man: Problems and Prospects, Proc. Intl. Bot. Conf., 10-12 Jan. 1991, pp. 47-53, Bangladesh Botanical Society, Dhaka.

Bold, H.C. and Wynne, M.J. 1985. Introduction to the Algae. 2nd edn. Prentice-Hall, New Jersey, pp. 1-706.

Desikachary, T.V. 1959. Cyanophyta. I.C.A.R., New Delhi, pp. 1-686.

Gerrath, J.F. and Denny, P. 1979. Fresh water algae of Sierra Leone. I. Euglenophyta. Nova Hedwigia. 31: 525-285.

Huber-Pestalozzi, G. 1983. Das Phytoplankton des Süsswassers. Systematik und Biologie. 7. Teil, 1. Hälfte, ord. Chlorococcales. E. Schweizerb. Verlagsb., Stuttgart, pp. 1-1044.

Hynes, H.B.N. and Pentelow, F.T.K. 1978. The Biology of Polluted Waters. Liverpool Univ. Press, pp. 1-202.

Islam, A.K.M. Nurul and Begum, Z.N.T. 1970. Studies on the phytoplanktons of Dacca district. Order: Chlorococcales. J. Asiatic Soc. Pak. 15(3): 227-271.

Islam, A.K.M. Nurul and Begum, Z.N.T. 1987. New records of algae of Bangladesh. III. Genus Pseudobohlinia (Chlorococcales). Bangladesh J. Bot. 16(1): 103-106.

Islam, A.K.M. Nurul and Irfanullah, H.M. 2005a. Hydrobiological studies within the tea gardens at Srimangal, Bangladesh. II. Algal flora (excluding Chlorophyceae). Bangladesh J. Plant Taxon. 12(1): 33-52. 
Islam, A.K.M. Nurul and Irfanullah, H.M. 2005b. Hydrobiological studies within the tea gardens at Srimangal, Bangladesh. III. Chlorophyceae (excluding desmids). Bangladesh J. Plant Taxon. 12(2): 1938.

Islam, A.K.M. Nurul and Irfanullah, H.M. 2005c. Hydrobiological studies within the tea gardens at Srimangal, Bangladesh. IV. Desmids (17 genera). Bangladesh J. Plant Taxon. 12(2): 49-62.

Islam, A.K.M. Nurul and Irfanullah, H.M. 2006. Hydrobiological studies within the tea gardens at Srimangal, Bangladesh. VI. Desmids (Xanthidium, Arthrodesmus, Staurodesmus and Staurastrum). Bangladesh J. Plant Taxon. 13(2): 111-129.

Islam, A.K.M. Nurul and Khatun, M. 1966. Preliminary studies on the phytoplanktons of polluted waters. Sci. Res. 3(2): 94-109.

Islam, A.K.M. Nurul and Nahar, L. 1967. Preliminary studies on the phytoplanktons of polluted waters. Part II. Blue-green algae. Sci. Res. 4(2\&3): 141-149.

Johansen, D. 1940. Plant Microtechnique. McGraw Hill Book Co. Inc., pp. 1-523.

Khondker, M., Bhuiyan, R.A., Yeasmin, J., Alam, M., Sach, R.B., Huq, A. and Colwell, R.R. 2006. New records of phytoplankton for Bangladesh. I. Cyanophyceae. Bangladesh J. Bot. 35(2): 173-180.

Khondker, M., Bhuiyan, R.A., Yeasmin, J., Alam, M., Sach, R.B., Huq, A. and Colwell, R.R. 2007a. New records of phytoplankton for Bangladesh. II. Cryptophyceae and Synurophyceae. Bangladesh J. Bot. 36(1): 53-60.

Khondker, M., Bhuiyan, R.A., Yeasmin, J., Alam, M., Sach, R.B., Huq, A. and Colwell, R.R. 2007b. New records of phytoplankton for Bangladesh. 4. Chlorococcales. Bangladesh J. Plant Taxon. 14(2): 83-92.

Khondker, M., Islam, A.K.M. Nurul, Begum, Z.N.T. and Haque, S. 1990. Limnological studies of four polluted ponds in and around Dhaka city with reference to indicator species. Bangladesh J. Bot. 19(1): 51-63.

Mohan, N. and Kumar, N. 1990. Influence of industrial effluents on blue-green algae. National symposium on Cyanobacterial nitrogen fixation. I.A.R.I., India. Jan. 29-31, 1990, p. 62.

Prescott, G.W. 1982 (reprinted). Algae of the Western Great Lakes Area. Otto Koeltz Sci. Publ., West Germany, pp. 1-977.

Round, F.E. 1985. The Ecology of Algae. Cambridge Univ. Press, Cambridge, pp. 1-653. 